\title{
Perceived needs in women with gestational diabetes: A qualitative study
}

Taraneh Emamgoli Khooshehchin ${ }^{1}$, Zohre Keshavarz², Maryam Afrakhteh ${ }^{3}$, Elham Shakibazadeh ${ }^{4}$, Soghrat Faghihzadeh $^{5}$

${ }^{1}$ Student Research Office, Ph.D. Candidate, Department of Reproductive Health, School of Nursing and Midwifery, International Branch, Shahid Beheshti University of Medical Sciences, Tehran, Iran

${ }^{2}$ Assistant Professor, Department of Reproductive Health, School of Nursing and Midwifery, Shahid Beheshti University of Medical Sciences, Tehran, Iran

${ }^{3}$ Department of Obstetrics and Gynecology of Shohada Tajresh Hospital, Shahid Beheshti University of Medical Sciences, Tehran, Iran

${ }^{4}$ Assistant Professor, Department of Health Education and Promotion, School of Public Health, Tehran University of Medical Sciences, Tehran, Iran

${ }^{5}$ Department of Biostatistics, Zanjan University of Medical Sciences, Zanjan, Iran

\section{Type of article: Original}

\begin{abstract}
Introduction: Diabetes is the most common medical complication of pregnancy. It can be associated with many complications for mother and fetus. Gestational diabetes is also one of the main health issues in Iran. Therefore, the present study is aimed at a deeper understanding of women's experiences of gestational diabetes and their perceived needs to inform future lifestyle interventions.

Methods: This qualitative content analysis study was carried out in 2015. Participants were pregnant women diagnosed with gestational diabetes in the $24^{\text {th }}$ to $36^{\text {th }}$ week of pregnancy, who were referred to the clinics affiliated with Shahid Beheshti Medical Science University in Tehran, Iran. In-depth interviews were conducted with participants, using semi-structured questions. Interviews were audio taped and transcribed verbatim. Conventional content analysis was carried out for data analysis. Interviews continued until data saturation was obtained. Data were coded in MAXQDA software (version 11).

Results: Content analysis highlighted two themes; educational needs and need to support. The former was featured with five main categories: information sources, education process, unknown and known, weaknesses of public information system, and eagerness to learn. The latter was featured with two main categories: family support and social support.

Conclusion: Clarifying the needs of the mothers with gestational diabetes, leads to better and proper education planning and a program toward the improvement of health, self-care, and prevention of diabetes.

Keywords: Diabetes, Pregnancy, Women, Needs, Content analysis
\end{abstract}

\section{Introduction}

Gestational diabetes is a glucose tolerance disorder, which is developed or diagnosed during the pregnancy period (1). The definition is regardless of whether insulin is used or not. Screening should be performed between 24 and 28 weeks of the gestational period in those women not known to have glucose intolerance earlier in pregnancy. This disease is a growing health problem all over the world and one of the most prevalent side-effects of pregnancy. In fact, prevalence of gestational diabetes had increased by $40 \%$ from 1989 to 2002. Prevalence of diabetes in different regions of the world varies from $1 \%$ to $14 \%$ (2). Prevalence of gestational diabetes in Iran is an estimated 4.9 percent. The lowest and the highest reported occurrences are in Kermanshah province $(0.7 \%)$ and in Karaj province

\section{Corresponding author:}

Assistant Professor Dr. Zohre Keshavarz, Department of Reproductive Health, School of Nursing and Midwifery, Shahid Beheshti University of Medical Sciences, Tehran, Iran.

Tel: +98.2188655366, Email: keshavarzzohre@yahoo.com and z.keshavarz@sbmu.ac.ir

Received: May 08, 2016, Accepted: July 13, 2016, Published: December 2016

iThenticate screening: July 07, 2016, English editing: September 20, 2016, Quality control: October 25, 2016

(C) 2016 The Authors. This is an open access article under the terms of the Creative Commons Attribution-NonCommercialNoDerivs License, which permits use and distribution in any medium, provided the original work is properly cited, the use is non-commercial and no modifications or adaptations are made. 
(18.6\%) respectively (3). Adverse maternal affects associated with gestational diabetes include an increased frequency of hypertension and cesarean delivery. Fetal and neonatal affects are perinatal morbidities such as birth injuries, hypoglycemia, future obesity, respiratory problems and stillbirth. Fasting hyperglycemia $>105 \mathrm{mg} / \mathrm{dL}$ may be associated with an increasing risk of fetal death during the final 4 to 8 weeks $(2,4,5)$. Gestational diabetes might be a sign of later development of diabetes type II in the mother and the child $(6,7)$. Development of gestational diabetes is related to the progressive history of diabetes type II in the family, the mother's age being more than 30 35 years old, overweight, ethnicity and race $(8,5)$. Gestational diabetes increases the risk of diabetes type 2 in women, which is preventable or can be delayed at least, by changing one's life style including diet and exercise. Therefore, we need to have an insight into the factors affecting diabetic women's behavior during and after delivery and the necessity of making proper interventions to avoid the disease, which also influences the wellbeing of the family (9). The majority of women do not attend pre-pregnancy care and do not have enough information about side-effects, self-care monitoring and management, or prevention, even when gestational diabetes is diagnosed. Regarding the cultural and social differences of different societies, the aim of this study was to explore the experiences of women with GDM, including factors associated with diabetes prevention practices, healthy behavioral change, and their perceptions regarding their health status, risk for diabetes and need for lifestyle modification, in order to plan more effective interventions to promote the health status of pregnant women. The objectives of this study were:

1) To describe facilitators and barriers that impact lifestyle modifications by women with gestational diabetes

2) To gain an understanding of the ongoing prenatal and postpartum needs of women with gestational diabetes

3) To discuss implications for best practices, health policies and future research concerning diabetes prevention in "at risk" women.

\section{Material and Methods}

\subsection{Study Design}

This was a qualitative study with the aim of deeper understanding and exploring women's experiences of gestational diabetes and their perceived needs, using a conventional content analysis approach.

\subsection{Study Location and Participants}

This research was a part of a larger mixed method study which was carried out in Iran during 2015. Participants consisted of 12 pregnant women diagnosed with gestational diabetes in $24^{\text {th }}-36^{\text {th }}$ week of pregnancy who were referred to the clinics affiliated with Shahid Beheshti Medical Science University. Participants were recruited by purposive sampling with maximum diversity in terms of age, occupation, education etc. In-depth interviews were conducted with participants, using semi-structured questions. Data saturation was obtained after 12 interviews.

\subsection{Study Procedures Data Collection}

Data was obtained from 12 semi-structured interviews. The participants were 12 pregnant women with gestational diabetes, who were recruited by purposive sampling with maximum diversity. Inclusion criteria were: pregnant women with diagnosis of gestational diabetes, with maximum diversity regarding their age, ethnicity, educational status, economic status, and without any history of clinical problems pre-pregnancy. Each woman was purposively recruited for this study. Each interview was conducted at a private and convenient location and took approximately 30 to 45 minutes. Interviews were audio taped and transcribed verbatim. Interviews continued until data saturation was obtained. The interview began with open-ended questions and continued with probing and follow up questions. As ideas emerged, the interviewer asked more focused questions. An occasional probe such as "can you tell me a little more about this" helped to elicit more information about the participants. The primary interview guide questions were:

1) Please explain how you felt when you were diagnosed with gestational diabetes?

2) What do you want to know about the GDM and where would you normally get your information from?

3) What are your needs during pregnancy as a diabetic mother?

4) What are your expectations during pregnancy with gestational diabetes?

5) Please explain any barriers affecting self-care in gestational diabetes?

\subsection{Data Analysis}

Data was analyzed using the conventional content analysis method. The qualitative content analysis is the analysis of narrative data to identify prominent themes and patterns among the themes. Interviews were completely audiotaped and transcribed verbatim. Data was coded in MAXQDA software (version 11). The condensed units were 
abstracted and labeled with codes. Then the codes were organized into subcategories and then the categories were derived, based on comparisons regarding their similarities and differences.

\subsection{Validity of the Study}

In order to ensure the credibility of research data, participants' checking was carried out. During the interview, the interviewee's words were fed-back to participants to confirm them. Furthermore, the codes extracted from the 4 interviews were shown to the respective participants and corrected under their supervision. Other methods such as prolonged engagement, sufficient time allocation and good communication were utilized. In order to reach transferability, detailed and comprehensive data description was applied so that other researchers could have a full understanding of research steps. In order to achieve conformability, two experts in the field of qualitative research and reproductive health were asked to review the reports and manuscripts, and present their findings. Very high similarity was found in the conclusions. In order to evaluate credibility, data was presented to an independent researcher and it was found that he concluded the same as the relevant researcher and as such, credibility and dependability of data was achieved.

\subsection{Ethical consideration}

The Research deputy and Ethics Committee of Nursing and Midwifery School of Shahid Beheshti University of Medical Sciences approved this study. The aim of the study was explained in detail to the participants. Informed consent was obtained from the participants in order to perform and record the interviews. The following information was given to the participants: the voluntary nature of the participation could withdraw from the study at any time they wished, as their right to privacy and confidentiality.

\section{Results}

A total of 12 pregnant women at an age range of $25-38$, and between $24^{\text {th }}-36^{\text {th }}$ week of pregnancy, without any history of diabetes or chronic diseases before pregnancy who participated in the study, received insulin or a special diet treatment. Those on insulin administered the medication themselves and they were compatible with their condition during the time of their pregnancy. In addition, $25 \%$ of the participants used insulin and a special diet concurrently. Content analyses highlighted two themes including educational needs and need to support. The former was comprised of main categories including: information resources, known and unknowns, weaknesses of public information system, and eagerness to learn. Needs to support included two main categories which were family support and social support (Table 1).

\subsection{The first main category: Educational Needs}

Subcategories of educational needs included sources of information, education process, knowns and unknowns, weakness of public information system, and eagerness to learn. The majority of the participants expressed that they had mostly relied on physicians, family, the mass media, and health personnel as their reliable sources of information. One pregnant woman stated: "My uncle's daughter in law said that they handled the problem using special diet. I followed their remedies, but I mostly followed the physician's orders" ( 26 year old, bachelors' degree, special diet). Another one mentioned: "A doctor in a program of Health Channel on TV said that diabetes is a serious disease if it is not controlled" (27 years old, bachelors' degree, diet). The majority of participants had different attitudes regarding the process of education such as: type, time, or method of education. Some preferred face-to-face education and some preferred pamphlets; and as argued by some of the participants, it ought to be provided in the early weeks of pregnancy or pre-pregnancy. One of them said: "It would be nice if they tell me what to do rather than just giving us a piece of paper as pamphlet" (30 years old, bachelors' degree, diet + insulin). "The doctors should educate their patients themselves, because all patients trust their own physicians" (26 years old, bachelors' degree, diet). Another one said: "Of course, if so, the physician must inform me about the side effects of pregnancy and what I should do." (27 years old, bachelors, diet). The majority of the participants mentioned that the health care provider just gave information about specific matters, such as side effects of diabetes during pregnancy. This kind of incomplete information may lead to excessive stress on the mother and affected their self-care behavior during pregnancy. A mother said: "Nope, they didn't say anything about what was going on; they just said, it is dangerous for your child." (34 years old, diploma, diet + insulin). Another one said: "I stopped eating bread and rice from the moment I learned about the bad news until I visited my doctor, who said that the child needs starch and I should take some." (26 years old, bachelors, diet). Another mother said: "I do not know what I should do; I take a blood sample every one or two hours before having breakfast. There is a laboratory near the place I live, I'll go there." (28 years old, elementary, diet). Some of the participants said that they do not have enough knowledge about diabetes and some said that they had received enough information. "I know how to control my blood sugar by diet in 
pregnancy; however, I received no education regarding after delivery. (25 years old, diploma, diet). "Some of the doctors said I'll be a diabetic by 40 for sure." (34 years old, diploma, insulin + diet). "Unfortunately, my physician says almost nothing; even if you ask a question, you will get the answer with a 2 minute delay. She is of no help." (27 years old, bachelors, diet). The majority of the participants expressed their desire to acquire more information about the disease's, side-effects, and progress. Few of them showed no interest in learning about their disease because of stresses and worries. A pregnant woman said: "Well, I'd like to know what is wrong with me and also what would be the side effects" ( 25 years old, diploma, diet). "I've read 10 to 20 books about delivery, diabetes and so on." (38 years old, master degree, diet). "My doctor gave me a book about gestational diabetes, but I didn't read even a single page; as they say, the less you know, the better you live." (26 years old, bachelor, diet).

Table 1. Perceived needs in women with gestational diabetes

\begin{tabular}{|c|c|c|c|}
\hline Theme & Main categories & subcategories & Codes \\
\hline \multirow{32}{*}{$\begin{array}{l}\text { Perceived } \\
\text { needs }\end{array}$} & \multirow{30}{*}{$\begin{array}{l}\text { Educational } \\
\text { needs }\end{array}$} & \multirow{3}{*}{$\begin{array}{l}\text { Information } \\
\text { sources }\end{array}$} & The physician and the staff's help \\
\hline & & & The help from family members, friends, and the elderly relatives. \\
\hline & & & Collecting information from the mass media \\
\hline & & \multirow{5}{*}{$\begin{array}{l}\text { Education } \\
\text { process }\end{array}$} & Importance and preference about type and method of education \\
\hline & & & Importance and preference about the educator \\
\hline & & & Importance and preference about the content of education \\
\hline & & & Importance and preference about place of education \\
\hline & & & Importance and preference about time of education \\
\hline & & \multirow{6}{*}{$\begin{array}{l}\text { known and } \\
\text { unknowns }\end{array}$} & Emergence and course of the disease \\
\hline & & & Follow up and control during pregnancy and after delivery \\
\hline & & & Side-effects (fetus and mother) \\
\hline & & & $\begin{array}{l}\text { The method of measuring blood sugar, insulin injection and using } \\
\text { glucometer }\end{array}$ \\
\hline & & & Treatment and self-care for gestational diabetes \\
\hline & & & Effective factors on creating and controlling gestational diabetes \\
\hline & & \multirow{8}{*}{$\begin{array}{l}\text { Weakness of } \\
\text { public } \\
\text { information } \\
\text { system }\end{array}$} & Provision or lack of information about after-delivery health cares \\
\hline & & & $\begin{array}{l}\text { Informing women about the relationship between higher age and } \\
\text { risk of diabetes }\end{array}$ \\
\hline & & & $\begin{array}{l}\text { Informing women about the relationship between being } \\
\text { overweight and higher risk of diabetes }\end{array}$ \\
\hline & & & $\begin{array}{l}\text { Provision or lack of information about recurrence of gestational } \\
\text { diabetes and future pregnancy }\end{array}$ \\
\hline & & & Lack of enough information about the course of the disease \\
\hline & & & Training insulin injection \\
\hline & & & $\begin{array}{l}\text { Provision or lack of information about the method of measuring } \\
\text { blood sugar and using glucometer }\end{array}$ \\
\hline & & & Provision of further information as a stressor \\
\hline & & \multirow{8}{*}{$\begin{array}{l}\text { Eagerness to } \\
\text { learn }\end{array}$} & Desire to know \\
\hline & & & Desire to learn information from others \\
\hline & & & Feeling no need for information from others \\
\hline & & & Feeling the need for extra education about insulin injection \\
\hline & & & $\begin{array}{l}\text { Relying on the education provided by the physicians and not } \\
\text { trying to find additional information }\end{array}$ \\
\hline & & & $\begin{array}{l}\text { Necessity of being informed before pregnancy and before } \\
\text { development of the disease }\end{array}$ \\
\hline & & & $\begin{array}{l}\text { Necessity of educations about the way and occasions of follow up } \\
\text { services }\end{array}$ \\
\hline & & & Necessity of education about type of follow up services \\
\hline & \multirow[t]{2}{*}{ Need to support } & $\begin{array}{l}\text { Family } \\
\text { support }\end{array}$ & Improving sense of solidarity and sympathy \\
\hline & & Social support & Physicians and personnel \\
\hline
\end{tabular}




\subsection{The second main category: Needs to support}

3.2.1. Subcategories of need to support include family support and social support.

Most of the participants mentioned that they received good support from a family member. "My husband is a great supporter and really helps me. He says never think about bad things and try to be optimistic." (30 years old, master degree, diet). Some of the diabetic mothers stated that they needed more support by their spouses. "In these stressful conditions, everyone likes to find their husband is supportive and sympathetic." (34 years old, diploma, diet + insulin).

\subsubsection{Social support}

Regarding the supporting role played by physicians and health care providers, some of the participants were satisfied and some were not. One pregnant woman stated: "Thank God, everyone was nice and helpful even when I was hospitalized." (34 years old, diploma, diet+ insulin). Another one mentioned: "I had bleeding in the first trimester and immediately went to the clinic, the doctor prescribed medication but carried out no test. He said, let's wait and see if the fetus survives. By then I had gestational diabetes for 2.5 months. The fetus' brain was developing during those 2.5 months, if my disease has affected the child's brain, who would be responsible for this." (37 years old, bachelors' degree, diet).

\section{Discussion}

This is the first study on the perceptions of women with gestational diabetes in Iran. The results of this study indicate that women with gestational diabetes have needs which were not fulfilled. Educational and support needs are two themes that were obtained from this study.

\subsection{Educational Needs}

Educational needs were frequently mentioned by the participants during the interviews. One of the main issues was a lack of enough knowledge about the course of the disease, self-care methods, and follow ups. Some of the participants were satisfied with information provided by the physicians and the health care providers, while the majority were not satisfied. Participants in this study mentioned that part of the information they received was provided by the physicians and the health care providers, and other parts were provided by their friends and relatives. The most critical information provided by the health care providers was about diet and how to do physical activities, which in some cases, the information was not complete. Devsam et al.' who conducted a study in Sweden found participants experienced a gap between the early diagnosis of the disease and the next examination two weeks later, during which they were not provided with enough information (10). The majority of the participants mentioned that being informed about the problem was important in controlling blood sugar levels (11). Goldstein et al. reported that the women who were admitted in to general ward were satisfied with the information they received about proper life style (29 out of $36-81 \%$ ) and treatment methods (26 out of 36- $72 \%$ ) (8). Hjelm et al. conducted a study in Sweden and reported that all of their subjects received information about gestational diabetes with delay (12). Another study by Van Ryswyk showed that being diagnosed with gestational diabetes induced anxiety and stress in mothers. Necessity of specific information about the disease was highlighted soon after the disease was diagnosed. Although, the majority of the women were informed about the risk of diabetes and prevention process, they reported many problems in performing the process. This means that they needed support for changing their life style by receiving prenatal health care. This study indicated that receiving education about gestational diabetes, prenatal health care after diagnosis, and follow up health care services were the reason that the women sought health services and medical attention (13). Greenhalgh et al. found that women's awareness about pre-gestational and gestational diabetes was at different levels. Some women had good knowledge about the diseases and preventive measures and some did not know the difference between diabetes type II and gestational diabetes (14). Meibodi et al. surveyed awareness and performance of pregnant women regarding gestational diabetes and reported that 659 women (95.9\%) had received information about the disease out of which 357 (35.7\%) received the information from clinics and $238(23.8 \%)$ from the mass media. Moreover, 648 patients $(64.8 \%)$ were able to give a correct definition about gestational diabetes and 757 patients $(75.7 \%)$ did not know that gestational diabetes might develop after the delivery. Additionally, 613 women (61.5\%) had records of diabetes in their family, 309 women (31.1\%) were pregnant several times, 581 women $(58.2 \%)$ were older than 30 years old, 690 women $(69.1 \%)$ suffered from obesity, and 582 women (58.2\%) suffered from high blood pressure and risk factors of gestational diabetes (15). Neufeld et al. reported that their subjects knew little about the diet (16). Devsam reported that their participants mentioned an unpleasant experience when the midwife, who informed them about the disease, did not tell them anything about the ways to control the disease or the side effects. Participants mentioned that they managed to cope with their problem, only when they gathered enough information via the Internet and books. They also mentioned that they felt better after they met an endocrinologist (10). The majority of the participants expressed their desire to 
receive information (were eager to learn) about diabetes and that they used different sources (the mass media, Friends and relatives who had experienced diabetes) to receive the information. Some of the participants mentioned that their family members' support was very effective on receiving information. Bieda argued in his study that the majority of gestational diabetic women were informed about the risks of diabetes type II and the preventive measures. Although the main source of information about the diseases was the family, the main information source about the risk of diabetes type II and the gestational diabetes were the experts and health care providers. In spite of problems and challenges, the participants had a high desire to participate in educational programs (17). Han reported that 11 participants $(50 \%)$ had searched for information about diabetes via the Internet $(\mathrm{n}=7)$, family and friends $(\mathrm{n}$ $=5)$, and experts $(\mathrm{n}=3)$. Four of those participants used more than one source (18). A 2012 study by Zalek et al showed that physicians' negligence regarding educating the patients about the disease, proper diet, and physical activities was mentioned by the participants that made them dissatisfied. In many cases, seeking information from people rather than health care providers resulted in wrong decisions (11). A systematic study by Van et al. showed that a majority of the participants had good knowledge about the risks of diabetes type II. Some of the participants received their information from health experts and some others mentioned the mass media as their source of information. They reported different attitudes regarding the risk of diabetes type II, which were functional of family records of the disease and other risk factors of diabetes type II (13). Results of this study highlighted obstacles in the way of finding information about gestational diabetes, including false self-confidence and not taking the problem seriously. None of the reviewed studies mentioned similar findings. In our study, one of the challenges in educational needs was that mothers believed gestational diabetes to be a temporary problem which would deplete after delivery. That is, they were not aware that gestational diabetes could lead to diabetes type II in future. Hjelm et al. showed that the women who were referred to the endocrinology clinic, were mostly worried about being afflicted with diabetes type II in the future. On the other hand, all the women who were referred to prenatal clinics believed that there were several factors effective on gestational diabetes and that it was a temporary problem. Their responses varied in a spectrum of knowing about the future risks of gestational diabetes, to lack of information about the side effects. They had more positive estimates about future risks of disease in comparison to the women who were referred to diabetes and endocrinology clinics (12).

\subsection{Support needs}

Another need that mothers expressed was the need of support. The majority of the participants in this study stated that they were supported by their family members and found this support effective on their ability to cope with their situation. Han et al. reported that family and friends' support was the only empowering social factor for performing health care measures $(n=8)$. The first requirement to overcome the problems, according to the participants, was life partner/parents' support (18). Devsam et al. mentioned the effective factors on the experience of mothers with gestational diabetes including the culture and beliefs, social stigma, social support, experts' support, receiving adequate and reliable information, social rights, and obstacles in the way of self-care. The family members and friends' support was effective on the participants' decision to choose healthier life styles. Support encourages the women to believe that they are in good hands, while inadequate support leads the pregnant women to feel more vulnerable as they feel that their friends and family members have deserted them (10). Short or long-term support is needed to implement changes in life style. Needs for support expressed by the participants were dissimilar, indicating that some emphasized on social support and the minority on financial support. Although, changes in life style and diet in particular were essential, keeping the changes and following the diet in the long term is not easy, and women usually need support in this regard (13). Bieda concluded that the family remained as the main source of information, motivation, and support to prevent diabetes, therefore, the critical role of the family must be taken into account, regarding preventive health behavior (17). Moreover, the minority of participants in the study said that the family support was not effective on all issues; so in some instances they had received wrong remedies. Carolan argued that some of the patients experienced hardships following the remedies prescribed by their family members. The author concluded that the mothers of the pregnant women had provided enough support and the subjects and their respective families had received proper education based on their culture and needs regarding the disease (5).

Results revealed that the role of health care providers is a critical role that is perceived as an important social support for pregnant women. Some of the participants in this study mentioned that they were not satisfied with measures taken by their physicians and the information provided, and expressed that they needed more social support from their health care providers and specialists. Bianca et al. argued that the support by the experts was effective on the subjects' experience. The patients, who were properly supported by the experts, felt that they were capable of changing and improving their life style. However, not all women had enough support from the experts. Some stated that they had received enough information from the health care provider and the experts; although the 
health care provider did not show sympathy and mental support. The patients also stated that keeping the diet without enough education was not easy (10). Kaptein et al. argued that women with gestational diabetes needed medical and social support to change their lifestyle and reduce the side effects of pregnancy and risk of diabetes in the future (19). Carolan maintained, according to his subjects' viewpoint, that emotional support was effective on reducing the anxiety and stress they felt, after being diagnosed with gestational diabetes. In addition, emotional support was helpful in performing daily health care, keeping the diet, measuring blood sugar, and doing exercise. The main support sources were 1. Spouse and family members; and 2- Experts. The spouse or life partner played the most critical role in providing support and motivating the patient to keep the diet (by having the same diet), to control weight, and carry out physical activities (by joining the patients in doing the exercises). Mothers and other family members of the pregnant women could be of great support, which is highly valuable when the pregnant woman is traumatized by learning about her disease. The participants who had no family support expressed that it was too hard to keep to the diet. Regarding the support by the experts and health care provider, the subjects had quite a different experience, and evaluated the support from excellent to unacceptable (5). Carcone reported that the experienced support from the family had significant relationship with diabetes type II (20). Elsenbruch et al. showed that lack of social support is an important prenatal risk factor and had detrimental effects on pregnancy outcome (21). Javid et al. conducted a quantitative study on diabetic pregnant women and found that women who experienced lower social support, have poor self-care, and have more stress. This indicates the necessity of paying more attention to self-care, and social support during pregnancy. An improvement in this regard can be achieved by increasing awareness, by educating pregnant women regarding a proper life style during pregnancy and ways to prevent gestational diabetes (22). Attarha et al. mentioned about the outcome of effective midwife-mother relationship and revealed that it can improve the quality of life of mothers and promote healthy behavior in mothers during pregnancy and after giving birth (23). Another study revealed that the majority of pregnant women were satisfied or very satisfied with the quality of care. All women received structured information from the health care team; which led to less fear and more positive thinking in diabetic mothers. This study concluded that the role of the health care team, especially for immigrant women with different languages, culture and religion is very important (24).

\section{Study Limitations}

The limitations of this study were to find and encourage participants with inclusion criteria, and collect data from pregnant mothers with gestational diabetes who were referred to clinics which took a long time for the researchers.

\section{Conclusions}

The results of this study revealed that participants expressed educational and support needs as the greatest problem. Therefore, by uncovering the perceived needs by mothers with gestational diabetes during pregnancy, the present paper is a great contribution to the design of a special education package for the target group. In addition, the results can be helpful to improve social support for pregnant women before and after delivery. Therefore performance and efficient intervention dependent on participants needs, may be helpful. As the findings indicated, it is essential to provide adequate support and education based on the culture and the perceived needs for the mothers with gestational diabetes and their family members.

\section{Acknowledgments:}

This article is a part of the $\mathrm{Ph}$.D. dissertation results of first author in reproductive health. The researchers appreciate all participants, personnel of health centers, and hospitals affiliated to the Medical Sciences Universities of Shahid Beheshti; without whose cooperation, this study would not have been possible.

\section{Conflict of Interest:}

There is no conflict of interest to be declared.

\section{Authors' contributions:}

All authors contributed to this project and article equally. All authors read and approved the final manuscript.

\section{References:}

1) Bandyopadhyay M, Small R, Mary-Davey A. Attendance for Postpartum Glucose Tolerance Testing Following Gestational Diabetes among South Asian Women in Australia: A Qualitative Study .J Womens Health Issues Care. 2015; 4(1): 1-8. doi: 10.4172/2325-9795.1000178. 
2) Cunningham FG, Leveno KJ, Bloom S, Spong CY, Dashe JS, Hoffman BL, et al. Williams obstetrics. New York. Mc Graw Hill. 2014; 1125-43.

3) Sayehmiri F, Bakhtiari S, darvishi P, Sayehmiri K. Prevalence of Gestational Diabetes Mellitus in Iran: A Systematic Review and Meta-Analysis Study. Iranian Journal of Obstetrics Gynecology and Infertility. 2013; 15(40): 23-16.

4) Metzger BE, Gabbe SG, Persson B, Lowe LP, Dyer AR, Oats JJN, et al. International association of diabetes and pregnancy study groups recommendations on the diagnosis and classification of hyperglycemia in pregnancy. Diabetes Care. 2010; 33(3): 676-82. doi: 10.2337/dc09-1848.

5) Carolan M. Women's experiences of gestational diabetes self-management :A qualitative study. Midwifery. 2013; 29(6): 637-45. doi: 10.1016/j.midw. 2012.05.013. PMID: 22877761.

6) Osgood ND, Dyck RF, Grassmann WK. The inter- and intragenerational impact of gestational diabetes on the epidemic of type 2 diabetes. Am J Public Health. 2011; 101(1): 173-9. doi: 10.2105/AJPH.2009.186890. PMID: 21148717, PMCID: PMC3000710.

7) Oster RT, Johnson JA, Hemmelgarn BR, King M, Balko SU, Svenson LW, et al. Recent epidemiologic trends of diabetes mellitus among status Aboriginal adults. CMAJ. 2011; 183(12): 803-8. doi: 10.1503/cmaj.101882.

8) Harizopoulou VC, Kritikos A, Papanikolaou Z, Saranti E, Vavilis D, Klonos E, et al. Maternal physical activity before and during early pregnancy as a risk factor for gestational diabetes mellitus. Acta Diabetol. (2010); 47(1): 83-89. doi: 10.1007/s00592-009-0136-1.

9) Parsons J, Ismail K, Amiel S, Forbes A. Perceptions Among Women With Gestational Diabetes. Qualitative health research. 2014; 24(4): 575-85. doi: 10.1177/1049732314524636.

10) Devsam BU, Bogossian FE, Peacoc AS. An interpretive review of women's experiences of gestational diabetes mellitus:Proposing a framework to enhance midwifery assessment. Women Birth. 2013; 26(2): 6976. doi: 10.1016/j.wombi.2012.12.003. PMID: 23333029.

11) Zalek K, Kazemi Hakami B, Motalebi H. Barriers to self care of patients with type 2 diabetes and ways to fix it. Journal of Student Research Committee Golestan University of Medical Sciences. 2013; 14(2): 30-7.

12) Hjelm K, Bard K, Berntorp K, Apelqvist J. Beliefs about health and illness postpartum in women born in Sweden and the Middle East. Midwifery. 2009; 25(5): 564-75. doi: 10.1016/j.midw.2007.08.007. PMID: 18206281.

13) Van Ryswyk E, Middleton P, Shute E, Hague W, Crowther C. Women's views and knowledge regarding healthcare seeking for gestational diabetes in the postpartum period: A systematic review of qualitative/survey studies. Diabetes Res Clin Pract. 2015; 110(2): 109-22. doi: 10.1016/j.diabres.2015.09.010. PMID: 26421361.

14) Greenhalgh T, Clinch M, Afsar N, Choudhury Y, Sudra R, Campbell-Richards D, et al. Socio-cultural influences on the behaviour of South Asian women with diabetes in pregnancy qualitative study using a multi-level theoretical approach. BMC Medicine. 2015; 13(120): 1-15. doi: 10.1186/s12916-015-0360-1.

15) Balali Mibodi, F, Mahmodi M, Hasani M. Knowledge, Attitude and Practice of Pregnant Women Referred to Health Care Centers of Kerman University of Medical Sciences in regard to Gestational Diabetes. Journal of qualitative Research in Health Sciences. 2011; 11(1-2): 17-24.

16) Neufeld HT. Food Perceptions and Concerns of Aboriginal Women Coping with Gestational Diabetes in Winnipeg, Manitoba. J Nutr Educ Behav. 2011; 43(6): 482-91. doi: 10.1016/j.jneb.2011.05.017. PMID: 22078771.

17) Bieda J. Percptions of risk for the development of type 2 diabetes in African-American women with gestational diabetes. Dissertation, The University of Michigan. 2009.

18) Han SF, Middleton PK, Bubner TA, Crowther C. Women's Views on Their Diagnosis and Management for Borderline Gestational Diabetes Mellitus. Journal of Diabetes Research. 2015; 2015: 1-9. doi: $10.1155 / 2015 / 209215$.

19) Kaptein S, Evans M, McTavish S, Banerjee AT, Feig DS, Lowe J, et al. The Subjective Impact of a Diagnosis of Gestational Diabetes Among Ethnically Diverse Pregnant Women: A Qualitative Study. Can J Diabetes. 2015; 39(2): 117-22. doi: 10.1016/j.jcjd.2014.09.005. PMID: 25512097.

20) Idalski Carcone A, Ellis DA, Weisz A, Naar-King S. Social support for diabetes illness management: Supporting adolescents and caregivers. J Dev Behav Pediatr. 2011; 32(8): 581-90. doi: 10.1097/DBP.0b013e31822c1a27. PMID: 21904213, PMCID: PMC3205417.

21) Elsenbruch S, Benson S, Rücke M, Rose M, Dudenhausen J, Pincus-Knackstedt MK, et al. Social support during pregnancy: effects on maternal depressive symptoms, smoking and pregnancy outcome. Hum Reprod. 2007; 22(3): 869-77. doi: 10.1093/humrep/del432. PMID: 17110400. 
22) Momeni Javid F, Simbar M, Dolatian M, Alavi Majd H. Comparison of Pregnancy Self-Care, Perceived Social Support and Perceived Stress of Women with Gestational Diabetes and Healthy Pregnant Women. Iranian Journal of Endocrinology and Metabolism. 2014; 16(3): 156-64.

23) Attarha M, Keshavarz Z, Bakhtiari M, Jamilian M. The Outcome of Midwife-Mother Relationship in Delivery Room: A Qualitative Content Analysis. Health. 2016; 8(4): 336-43. doi: 10.4236/health.2016.84035.

24) Lapolla A, Cianni G, Benedetto A, Franzetti I, Napoli A, Sciacca L, et al. Quality of Life, Wishes, and Needs in Women with Gestational Diabetes: Italian DAWN Pregnancy Study. International Journal of Endocrinology. 2012; (2012): 6. doi: 10.1155/2012/784726. 